Research Paper

\title{
Efficacy of Cabazitaxel Treatment in Metastatic Castration Resistant Prostate Cancer in Second and Later Lines. An Experience from Two German Centers
}

\author{
Stefanie Zschäbitz ${ }^{1}$, Sonia Vallet ${ }^{1}$, Boris Hadaschik ${ }^{2}$, Daniel Debatin ${ }^{3}$, Stefan Fuxius ${ }^{3}$, Andreas Karcher ${ }^{3}$, \\ Sascha Pahernik 2,4 , Cathleen Spath ${ }^{1,5}$, Stefan Duensing, 2,5 , Dirk Jäger ${ }^{1}$, Markus Hohenfellner ${ }^{2}$, Carsten \\ Grüllich ${ }^{1 凶}$ \\ 1. Department of Medical Oncology, National Center for Tumor Diseases (NCT), Heidelberg University Hospital, Im Neuenheimer Feld 460, 69120 \\ Heidelberg, Germany; \\ 2. Department of Urology, Heidelberg University Hospital, Im Neuenheimer Feld 110, 69120 Heidelberg, Germany; \\ 3. Onkologische Schwerpunktpraxis Heidelberg, Kurfürstenanlage 34, 69115 Heidelberg, Germany; \\ 4. Department of Urology, Klinikum Nürnberg, Paracelsus Medical University, Prof.-Ernst-Nathan-Str. 1, 90419 Nürnberg, Germany; \\ 5. Section of Molecular Urooncology, Department of Urology, Heidelberg University Hospital, Im Neuenheimer Feld 110, 69120 Heidelberg, Germany.
}

$\square$ Corresponding author: Carsten Grüllich, MD, PhD, Department of Medical Oncology, National Center for Tumor Diseases (NCT), Heidelberg University Hospital, Im Neuenheimer Feld 460,69120 Heidelberg, Germany. Phone: $\quad+496221$ 5637125; Fax: $\quad+49 \quad 6221 \quad 565318$; $\quad$ E-Mail: carsten.gruellich@med.uni-heidelberg.de.

( ) Ivyspring International Publisher. This is an open access article distributed under the terms of the Creative Commons Attribution (CC BY-NC) license (https://creativecommons.org/licenses/by-nc/4.0/). See http://ivyspring.com/terms for full terms and conditions.

Received: 2016.09.20; Accepted: 2016.12.23; Published: 2017.02.11

\begin{abstract}
Purpose: Several new treatment options for patients with metastatic castration resistant prostate cancer (mCRPC) have been approved within the last years - among them cabazitaxel (CAB), abiraterone acetate, enzalutamide, and radium-223. The aim of this study was to assess factors predictive for efficacy of CAB.

Methods: We analyzed all patients with $m C R P C$ treated with $C A B$ at our institutions between 2011 and 2016. Data were retrieved retrospectively from the electronical patient chart.

Results: 69 patients received CAB $\left(26.1 \% 2^{\text {nd }}\right.$ line, $36.2 \% 3^{\text {rd }}$ line, $37.3 \%>3^{\text {rd }}$ line $)$. Median overall survival (OS) on $C A B$ was 10.0 months $(95 \% \mathrm{Cl} 7.1-12.9)$. Median progression free survival (PFS) on $\mathrm{CAB}$ was 3.9 months $(95 \% \mathrm{Cl} 3.0-4.8)$. There were no differences in OS and PFS regarding treatment line of $C A B\left(2^{\text {nd }}\right.$ vs. higher; $2^{\text {nd }} / 3^{\text {rd }}$ vs. higher $)$. Duration of remission on $1^{\text {st }}$ line treatment ( $>6$ months vs. $</=6$ months) was associated with a longer PFS with subsequent $C A B$ treatment (4.1 months vs. 3.0 months $(95 \% \mathrm{Cl} 3.0-5.2 ; 2.2-3.8) ; \mathrm{p}=0.021)$. Patients with visceral metastases had a shorter PFS (3.0 months; $95 \% \mathrm{Cl} 2.6-3.3)$ and OS (8.7 months; $95 \% \mathrm{Cl} 5.9-11.5)$ on $\mathrm{CAB}$ compared to patients who had bone and/or lymph node lesions only (PFS: 5.8 months; $95 \% \mathrm{Cl}$ 3.2-8.4; $\mathrm{p}=0.014$; OS: 11.7 months; $95 \% \mathrm{Cl}$ 7.5-15.9; $\mathrm{p}=0.042$ ).

Conclusions: Results from our patient cohort suggest that a longer PFS to any $1^{\text {st }}$ line treatment for $\mathrm{mCRPC}$ is correlated with a longer PFS to $C A B$ for any later line treatment. Patients with nodal and bone metastases only had a significantly superior PFS and OS with CAB treatment than patients with visceral metastases.
\end{abstract}

Key words: Cabazitaxel, prostate cancer, sequencing therapy, survival, castration resistance.

\section{Purpose}

Patients that suffer from metastatic castration resistant prostate cancer (mCRPC) had few treatment options for a long time [1]. During the last decade major progress has been made extending the treatment options considerably. New generation anti-hormonal agents (NAA; abiraterone (ABI), 
enzalutamide (ENZA)) new radiotherapeutics (alpharadin; radium-223) and a new taxan chemotherapy (cabazitaxel (CAB)) have all been approved and shown to be active after failure of docetaxel (DOC) as a $2^{\text {nd }}$ line therapy $[2,3,4,5,6]$. However, limited data are available to differentiate patients that may benefit rather from chemotherapy with CAB than from a NAA post DOC. Moreover, none of these substances have been investigated in $3^{\text {rd }}$ or $4^{\text {th }}$ line within a prospective randomized clinical trial, and there is concern regarding acquired cross resistances among them. Nevertheless, in real-world oncology many patients receive more than two treatment lines aiming at a cumulative survival benefit that has yet to be confirmed. Prospective trials with approved substances are rarely ever conducted. Hence, retrospective analyses might help to improve decision making and treatment processes for this relevant patient group.

The objective of this study was to analyze all patients from our comprehensive cancer center and private practice who received $C A B$ either in $2^{\text {nd }}$ line or in later lines for clinical parameters associated with a response to $\mathrm{CAB}$.

\section{Methods}

In this retrospective observational study all 69 patients with mCRPC treated with $\mathrm{CAB}$ at the National Center of Tumor Diseases (NCT) and a private practice were identified and their records accessed through the electronic patients charts [7]. The project was approved by the local ethics committee.

Clinical parameters assessed included Gleason score (GS), tumor stage, location of metastases, time to castration resistance on androgen ablation therapy, type of $1^{\text {st }}$ line therapy, type of $2^{\text {nd }}$ line therapy, response to $1^{\text {st }}$ line therapy and duration of response (prostate specific antigen (PSA) and radiographic) as well as response and duration of response to later lines of CAB.

Progression free survival (PFS) was evaluated using the Prostate Cancer Working Group 2 (PCWG2) criteria [8]. Side effects were classified according to CTCAE dictionary version 4.0. Overall survival (OS) was calculated from the date of start of systemic anti-hormonal treatment and the start of $C A B$ treatment to the date of death or date of last follow-up (last assessed on March 2016). Survival and progression were calculated using Kaplan-Meier estimates and compared using log-rank tests. Associations between factors were evaluated using non-parametric Kendall-tau or Pearson correlation. Primary outcomes were the OS as well as the PFS since initiation of CAB. Secondary outcomes were PFS on $1^{\text {st }}$ line treatment as well as correlation analyses between treatment factors. Statistical analyses were conducted using the SPSS v21 software.

\section{Results}

The final study population consisted of 69 patients that started systemic anti-hormonal LHRH analogue treatment between February 1998 and January 2015. Most of the patients received DOC as $1^{\text {st }}$ line therapy $(n=52,75.4 \%)$. Other $1^{\text {st }}$ line therapies included ABI $(n=9)$, ENZA $(n=4)$, ipilimumab $(n=1$, as part of a clinical trial), and PSMA ligands $(n=3$, as part of a clinical trial). In $2^{\text {nd }}$ line, patients received $A B I$ $(n=31)$, CAB $(n=18)$, DOC $(n=12)$, ENZA $(n=5)$, RNA vaccine $(n=1$, as part of a clinical trial), and mitoxantrone $(n=2)$. $C A B$ was applied as $3^{\text {rd }}$ line treatment in 25 patients, as $4^{\text {th }}$ line therapy in 19 patients, as $5^{\text {th }}$ line in 5 patients, and as $6^{\text {th }}$ line in 2 patients.

$\mathrm{CAB}$ was administered according to the protocol that was established by the Phase-III TROPIC trial in the majority of patients [1]. However, $17.3 \%$ of patients received an upfront dose-reduction (i.e. $20 \%$ $=20 \mathrm{mg} / \mathrm{m}^{2} \mathrm{qd} 22, \mathrm{n}=9 ; \sim 30 \%=17.5-18 \mathrm{mg} / \mathrm{m}^{2} \mathrm{qd} 22$, $\mathrm{n}=3$ ) due to reduced general condition (ECOG 2 or ECOG 1 plus bone marrow dysfunction). Three patients were treated biweekly analogue the Prosty II trial protocol [9]. Six patients received $\mathrm{CAB}$ on a weekly scheme. In four cases dose reductions were necessary after cycles $1(n=2), 2(n=1)$ and $6(n=1)$ due to bicytopenia, respectively. All patients received $\mathrm{CAB}$ in $4^{\text {th }}$ line. In 3 patients, $\mathrm{CAB}$ regime was changed from three-weekly to weekly after cycle 1 due to bicytopenia. Those patients had received $C A B$ in $3^{\text {rd }}, 4^{\text {th }}$ and 6 th treatment line. Treatment was generally well tolerated with an average of 5 cycles (range 1-11) and a treatment duration of 3.6 months (range 0.4-17.0). Treatment was discontinued in most cases either due to progressive disease or when anticipated cycles of $C A B$ had been applied. In one case $C A B$ treatment was terminated due to patient preference. In four patients treatment was discontinued due to toxicity (neutropenic fever CTC $4^{\circ}(\mathrm{n}=2)$; acute renal insufficiency CTC $4^{\circ}(\mathrm{n}=1)$; peripheral sensory and motor neuropathy CTC $3^{\circ}$ in an 88 -years old individual $(\mathrm{n}=1)$ ). Three $\mathrm{CTC} 5^{\circ}$ side effects were noted: Two patients died in neutropenic sepsis (84-years old patient on cycle 1 day 11; 75-years old patient on cycle 2 day 11). A 71-years old patient died due to spontaneous subdural hematoma as a result of CAB-induced thrombocytopenia. Three patients are still on treatment. Patient characteristics are summarized in Table 1.

Median time between the diagnosis of prostate cancer and the initiation of androgen deprivation was 
2.0 months (95\% CI 1.0-7.9). Median time to castration resistance was 35.0 months for all investigated patients (95\% CI 26.0-43.0, Fig. 1a). Median PFS on $1^{\text {st }}$ line treatment was 7.0 months $(95 \%$ CI 5.5-8.5, Fig. $1 \mathrm{~b})$. There were no significant differences regarding PFS on $1^{\text {st }}$ line treatment between DOC vs. any other treatment type or GS 6-7 vs. 8-10.

Table 1. Patient Characteristics.

\begin{tabular}{|c|c|c|}
\hline Parameter & No. & \% (Range) \\
\hline Total number of patients & 69 & 100 \\
\hline Age at diagnosis [years], median & 62 & $(46-81)$ \\
\hline Age at CAB [years], median & 69 & $(51-88)$ \\
\hline \multicolumn{3}{|l|}{ Gleason Score at first diagnosis } \\
\hline $6-7$ & 27 & 39.1 \\
\hline $8-10$ & 42 & 60.9 \\
\hline \multicolumn{3}{|l|}{ Clinical nodal status at first diagnosis } \\
\hline No & 32 & 46,4 \\
\hline N1 & 33 & 47,8 \\
\hline missing & 4 & 5,8 \\
\hline \multicolumn{3}{|l|}{ Metastatic spread (prior to CAB treatment) } \\
\hline bone metastases & 59 & 85.5 \\
\hline peritoneal metastases & 4 & 5.8 \\
\hline lung metastases & 14 & 20.3 \\
\hline liver metastases & 24 & 34.8 \\
\hline brain metastases & 3 & 4.3 \\
\hline adrenal gland metastases & 8 & 11.6 \\
\hline CAB treatment cycles, median & 5 & $(1-11)$ \\
\hline Duration on $\mathrm{CAB}$, median & 3.6 & $(0.4-17.0)$ \\
\hline \multicolumn{3}{|l|}{ CAB regime } \\
\hline $25 \mathrm{mg} / \mathrm{m}^{2}, \mathrm{qd} 21$ & 42 & 60.8 \\
\hline $20 \mathrm{mg} / \mathrm{m}^{2}, \mathrm{qd} 21$ & 9 & 13.0 \\
\hline $17.5-18.0 \mathrm{mg} / \mathrm{m}^{2}, \mathrm{qd} 21$ & 3 & 4.3 \\
\hline $16 \mathrm{mg} / \mathrm{m}^{2}, \mathrm{qd} 15$ & 3 & 4.3 \\
\hline $10 \mathrm{mg} / \mathrm{m}^{2}, \mathrm{qd} 8$ & 6 & 8.7 \\
\hline unknown & 6 & 8.7 \\
\hline \multicolumn{3}{|l|}{ Type of first line treatment } \\
\hline Docetaxel & 52 & 75.4 \\
\hline Abiraterone & 9 & 13.0 \\
\hline Enzalutamid* & 4 & 5.8 \\
\hline PSMA ligand* & 3 & 4.3 \\
\hline Ipilimumab (within clinical trial) & 1 & 1.4 \\
\hline \multicolumn{3}{|l|}{ Type of 2nd line treatment } \\
\hline Docetaxel & 12 & 17.4 \\
\hline Abiraterone & 31 & 44.9 \\
\hline Enzalutamid & 5 & 7.2 \\
\hline $\mathrm{CAB}$ & 18 & 26.1 \\
\hline Mitoxantron & 2 & 2.9 \\
\hline RNA vaccine* (within clinical trial) & 1 & 1.1 \\
\hline \multicolumn{3}{|l|}{ CAB sequence } \\
\hline $2^{\text {nd }}$ line & 18 & 26.1 \\
\hline $3^{\text {rd }}$ line & 25 & 36.2 \\
\hline $4^{\text {th }}$ line & 19 & 27.5 \\
\hline $5^{\text {th }}$ line & 5 & 7.2 \\
\hline $6^{\text {th }}$ line & 2 & 2.9 \\
\hline Mean PSA at initial diagnosis & $96.2 \mathrm{ng} / \mathrm{ml}$ & $(0.1-900.0)$ \\
\hline Mean PSA at time point of castration resistance & $68.5 \mathrm{ng} / \mathrm{ml}$ & $(0.6-355.0)$ \\
\hline Mean PSA at time point of CAB begin & $453.0 \mathrm{ng} / \mathrm{ml}$ & $(0.0-5102.0)$ \\
\hline $\begin{array}{l}\text { Mean PSA at time point of progressive disease } \\
\text { post-CAB }\end{array}$ & $478.8 \mathrm{ng} / \mathrm{ml}$ & $(0.0-5074.0)$ \\
\hline \multicolumn{3}{|l|}{ PSA response in treatment period } \\
\hline$\geq 30 \%$ & 24 & 34.8 \\
\hline$\geq 50 \%$ & 20 & 29.0 \\
\hline$\geq 90 \%$ & 4 & 5.8 \\
\hline
\end{tabular}

Abbreviations: CAB: cabazitaxel; PSA: prostate specific antigen; PSMA: prostate specific membrane antigen.
Median OS since start of CAB treatment was 10.0 months (95\% CI 7.0-12.9). Median PFS on CAB treatment was 3.9 months (95\% CI 3.0-4.8, Fig. 2a). There were no differences on OS and PFS on CAB stratified for GS (6-7 vs. 8-10), age at diagnosis, age at begin of $C A B$ treatment, type of $1^{\text {st }}$ line therapy (DOC vs. any other), line of $\mathrm{CAB}\left(2^{\text {nd }}\right.$ vs. any other; $2^{\text {nd }} / 3^{\text {rd }}$ vs. any other), and absolute PSA prior to $C A B$ treatment (analyzed for percentile ranks 50; 66.7; 75) (data not shown). A PSA decline $\geq 50 \%$ was seen in $\mathrm{n}=20$ patients receiving CAB $(29.0 \%)$. A PSA reduction of at least $30 \%$ was noticed in $n=24$ patients $(24.8 \%)$. PSA decline $\geq 50 \%$ on $\mathrm{CAB}$ showed a prolonged OS (16.6 months vs. 9.2 months; $95 \%$ CI 6.0-27.3; 6.2-12.2; $\mathrm{p}=0.087)$ and PFS (6.9 vs. 3.0 months, 95\% CI 3.7- 10.2; 2.7-3.3; $\mathrm{p}=0.09)$, however not statistically significant. Statistical significance was reached for the larger cohort with a PSA decline of at least $30 \%$ for OS (16.6 vs. 8.8 months; $95 \%$ CI 6.5-26.7; 6.2-11.4; $\mathrm{p}=0.039)$ and PFS (6.9 vs. 3.0 months; $95 \% \mathrm{CI}$ 4.5-9.3; 2.7-3.3; $\mathrm{p}=0.03$ ).

When stratified for PFS on $1^{\text {st }}$ line therapy $(\leq 6$ months vs. $>6$ months), patients with a PFS $>6$ months had a significantly longer PFS on CAB in any later treatment line that was 4.1 months vs. 3.0 months (95\%CI 3.0-5.2; 2.2-3.8; p=0.021, Fig. 2b). No associations were noted between OS and duration of remission on $1^{\text {st }}$ treatment line. However, there was a trend towards longer $\mathrm{OS}$ on $\mathrm{CAB}$ treatment for patients that remained on remission on DOC $>6$ months (10.6 vs. 7.6 months; $95 \%$ CI 7.8-13.4; 4.4-10.8; $\mathrm{p}=0.051)$.

Patients with visceral metastases at time of $\mathrm{CAB}$ initiation had a significantly shorter PFS on $\mathrm{CAB}$ reaching 3.0 months vs. 5.8 months $(95 \% \mathrm{CI}$ 2.7-3.3; 3.2-8.4; $\mathrm{p}=0.014$, Fig. 2c) and a shorter OS compared to those who only had bone or/and lymph node lesions (8.7 months vs. 11.7 months, 95\% CI 5.9-11.5; 7.5-15.9; $\mathrm{p}=0.042$, Fig. $2 \mathrm{~d}$ ) whereas PFS on $1^{\text {st }}$ line treatment did not differ significantly between those two groups.

\section{Discussion}

Therapeutic options for MCRPC patients have been greatly expanded within the last couple of years. Beside NAAs, with CAB a novel taxane has also entered clinical practice [10,11]. $\mathrm{CAB}$ has been approved for the treatment of $\mathrm{MCRPC}$ in the post-docetaxel setting as a $2^{\text {nd }}$ line treatment and is currently investigated within the FIRSTANA trial as $1^{\text {st }}$ line treatment option at different dose levels. However, in the real world $C A B$ is often also used post $\mathrm{ABI}$ or ENZA as a $3^{\text {rd }}$ or $4^{\text {th }}$ line therapy being efficient in those settings $[12,13,14,15]$. Retrospective single and multicenter experiences as well as meta-analyses have come to contradicting results for 
the optimal sequence of medication. While some showed no significant differences between the different agents used in $3^{\text {rd }}$ or $4^{\text {th }}$ line [16], others pointed to a survival benefit when applying the sequence DOC-CAB-ABI $[14,17,18]$ or the sequence of CAB-NAA or vice versa compared to NAA-NAA post DOC [19]. However, no prospective randomized clinical trial has investigated treatment outcomes beyond $2^{\text {nd }}$ line and for different sequences. In addition, in the aftermath of the CHAARTED and STAMPEDE trials more and more patients will have

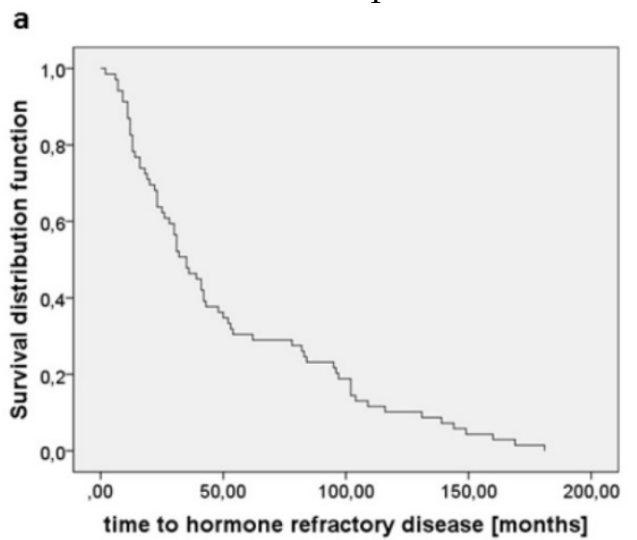

received DOC in the hormone-sensitive setting raising new questions of optimal therapeutic approaches upon the development of castration resistant disease. In our retrospective cohort patients with a PFS longer than 6 months on $1^{\text {st }}$ line therapy had a significantly longer PFS on CAB irrespective of the line of treatment. Interestingly, it has been shown before, that cross resistance may exist between new anti-androgens and taxanes which is in accordance with our data $[20,21]$.

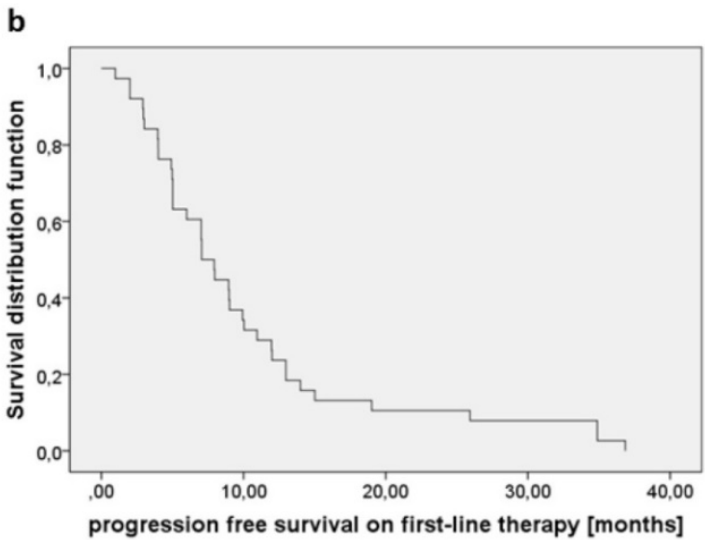

Figure 1. a) Time between initiation of systemic LHRH treatment and the development of castration resistant disease was 35.0 months. b) PFS on 1st line treatment independent of treatment type was 7.0 months.
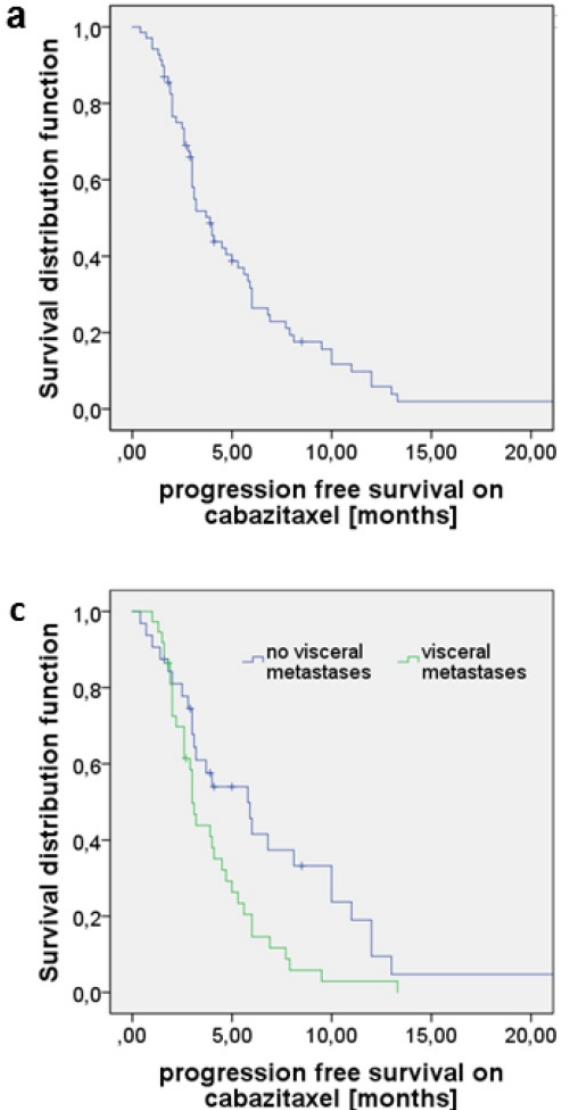
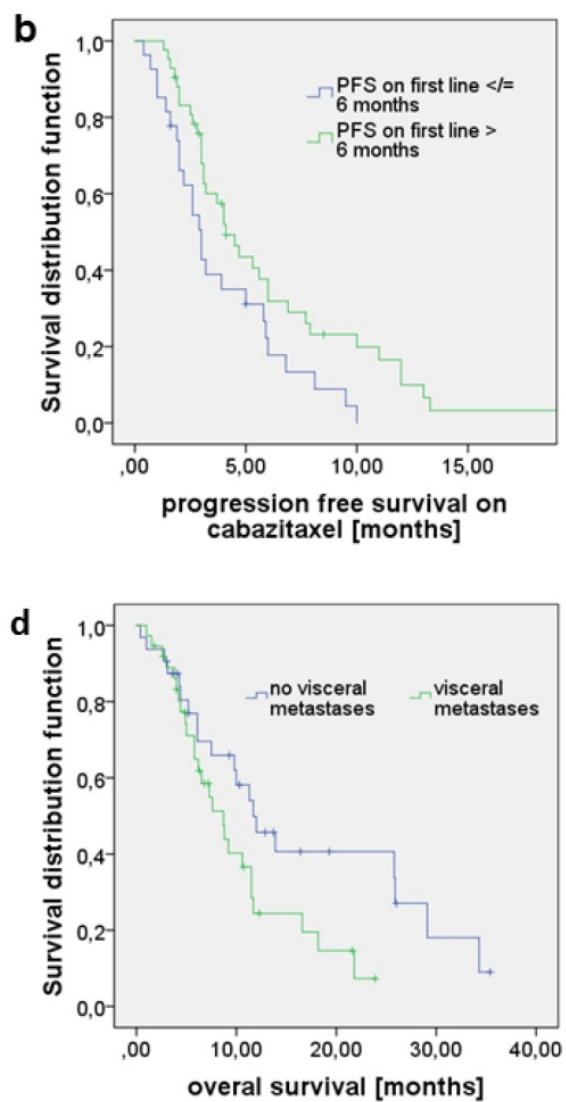

Figure 2. a) Progression free survival (PFS) on cabazitaxel (CAB) as $2^{\text {nd }}$ or later line treatment; median PFS was 3.9 months. b) PFS on CAB stratified for PFS on 1 st line ( $<6$ months vs. $>6$ months). Patients with $P P_{1 s t}$ line $>6$ months had a significantly longer $P F S$ on $C A B$ in any later treatment line $(4.1$ months vs. 3.0 ; $p=0.021)$. c) PFS of patients with visceral metastases was 3.0 compared to 5.8 months when no visceral metastases were present $(p=0.014)$. $d)$ Overall survival $(O S)$ of patients with visceral metastases was 8.7 compared to 11.7 months in the absence of visceral metastases $(p=0.042)$. 
A high GS (as defined as 8-10) was not predictive for response to $\mathrm{CAB}$ as previously reported [22]. While previously published studies showed a correlation between longer duration of remission on first line DOC for higher GS [21,23] we could not detect any such correlation for $\mathrm{CAB}$ in our cohort. We further found no correlation between the absolute level of PSA pre-CAB. While PSA increases during treatment for CRPC may be flare up phenomena and are not generally associated with radiographic progression [4], a PSA decline is in most studies associated with a better survival. We could also demonstrate that statistical significance for OS benefit was already reached with a PSA decline of at least $30 \%$.

Large pooled retrospective analyses have revealed that the existence of visceral metastases is a negative prognostic factor associated with reduced OS of 13.3 months for patients with liver metastases and 19.4 months for patients with lung metastases $[24,25]$. In our cohort, OS on CAB for patients with all types of visceral metastases was 8.7 months only. Interestingly, we also noted a significant difference in PFS on CAB in favor for patients with bone and/or bone and lymph node metastases compared to those with visceral metastases in our cohort (5.8 vs. 3.0 months). A difference in PFS was not observed in $1^{\text {st }}$ line treatment when stratified for visceral metastases. One explanation for the poor response of patients with visceral metastases could be that clonal selection associated with visceral spreading is associated with a more therapy-resistant phenotype, e. $g$ neuroendocrine subtypes [26,27]. Biopsies from visceral metastatic sites might help to further genetically characterize these clones and search for other treatment protocols. More prospective data are required to determine if $\mathrm{CAB}$ is a meaningful choice for $\mathrm{mCRPC}$ with visceral involvement.

We reported three grade 5 toxicities and several treatment discontinuations and dose modifications showing that $C A B$ has substantial toxicity in this elderly and frail population. It should be noted that lower doses are equally effective with lower toxicity $[28,29]$ and should be considered as initial dose level.

Limitations to our study are its retrospective character, dual center data and small sample size that may have introduced a bias. These factors might explain the inability to detect survival differences due to to inadequate power in some analyses.

\section{Clinical Practice Points}

The optimal treatment sequence for agents in mCRPC is still unknown. Prospective clinical trials are needed.

\section{Conclusions}

Sequencing of new substances for mCRPC remains a subject of debate. $C A B$ is approved for second line therapy following DOC. However, it is also used following new anti-hormonal treatments or in later lines. In our analysis CAB seems to be active in all these indications, especially if the tumor was sensitive to any $1^{\text {st }}$ line treatment, irrespective of the line of treatment when $\mathrm{CAB}$ was being deployed. However, patients with visceral metastases had a significantly shorter OS and PFS on CAB. Prospective studies are needed to identify the optimal treatment sequence for patients with mCRPC.

\section{Abbreviations}

ABI: abiraterone; CAB: cabazitaxel; DOC: docetaxel; ENZA: enzalutamide; GS: Gleason score; mCRPC: metastatic castration resistant prostate cancer; NAA: new generation anti-hormonal agent; OS: overall survival; PFS: progression free survival; PSA: prostate specific antigen; PSMA: prostate specific membrane antigen

\section{Acknowledgments}

The NCT is supported by the German Cancer Research Center (DKFZ), the University Hospital Heidelberg in cooperation with the Medical Faculty Heidelberg and by the German Cancer Aid (Deutsche Krebshilfe). We thank the NCT registry for providing information on survival dates.

\section{Authors' contributions}

D Debatin: Data collection

S Duensing: Data collection

S Fuxius: Data collection

C Grüllich: Protocol/project development, Data collection, Data analysis, Manuscript writing/editing

B Hadaschik: Data collection

M Hohenfellner: Data collection

D Jäger: Data collection

A Karcher: Data collection

S Pahernik: Data collection

C Spath: Data collection

$S$ Vallet: Data collection, Data analysis

S Zschäbitz: Protocol/project development, Data collection, Data analysis, Manuscript writing

\section{Ethics Committee Approval and Patient Consent}

All procedures performed in studies involving human participants were in accordance with the ethical standards of the institutional and/or national research committee and with the 1964 Helsinki declaration and its later amendments or comparable ethical standards. All patients provided written 
informed consent. Any information connected with the identity of individual subjects was excluded from this study.

Consent for publication was approved by the local ethics committee. All authors have read and agreed on the current version of the manuscript.

\section{Availability of data and material}

Original data and material are completely available upon requested from the corresponding author.

\section{Competing Interests}

The authors have declared that no competing interest exists.

\section{References}

1. Tannock IF, de Wit R, Berry WR, et al. Docetaxel plus prednisone or mitoxantrone plus prednisone for advanced prostate cancer. N Engl J Med. 2004; 351: 1502-1512.

2. de Bono JS, Oudard S, Ozguroglu M, et al. Prednisone plus cabazitaxel or mitoxantrone for metastatic castration-resistant prostate cancer progressing after docetaxel treatment: a randomised open-label trial. Lancet. 2010; 376: 1147-1154.

3. de Bono JS, Logothetis CJ, Molina A, et al. Abiraterone and increased survival in metastatic prostate cancer. N Engl J Med. 2011; 364: 1995-2005.

4. Fizazi K, Scher HI, Molina A, et al. Abiraterone acetate for treatment of metastatic castration-resistant prostate cancer: final overall survival analysis of the COU-AA-301 randomised, double-blind, placebo-controlled phase 3 study. Lancet Oncol. 2012; 13: 983-992.

5. Scher HI, Fizazi K, Saad F, et al. Increased survival with enzalutamide in prostate cancer after chemotherapy. N Engl J Med. 2012; 367: 1187-1197.

6. Parker C, Nilsson S, Heinrich D, et al. Alpha emitter radium-223 and survival in metastatic prostate cancer. N Engl J Med. 2013; 369: 213-223.

7. Huber J, Herpel E, Jakobi H, et al. Two decades' experience with a prospective biobank for urologic oncology: research, clinical care, and the patients' view. Urol Oncol. 2013; 31: 990-996.

8. Scher HI, Halabi S, Tannock I, et al. Design and end points of clinical trials for patients with progressive prostate cancer and castrate levels of testosterone: recommendations of the Prostate Cancer Clinical Trials Working Group. J Clin Oncol. 2008; 26: 1148-1159.

9. Lehtinen P-LIK. Biweekly cabazitaxel as post-docetaxel treatment for metastatic castration resistant prostate cancer (mCRPC): Findings from an early safety analysis of the Prosty II trial. J Clin Oncol. 2015; 33.

10. Omlin A, Pezaro CI, Zaidi S, et al. Antitumour activity of abiraterone and diethylstilboestrol when administered sequentially to men with castration-resistant prostate cancer. Br J Cancer. 2013; 109: 1079-1084.

11. Bianchini D, Lorente D, Rodriguez-Vida A, et al. Antitumour activity of enzalutamide (MDV3100) in patients with metastatic castration-resistant prostate cancer (CRPC) pre-treated with docetaxel and abiraterone. Eur J Cancer. 2014; 50: 78-84

12. Sella A, Sella T, Peer A, et al. Activity of cabazitaxel after docetaxel and abiraterone acetate therapy in patients with castration-resistant prostate cancer. Clin Genitourin Cancer. 2014; 12: 428-432.

13. Pezaro CJ, Omlin AG, Altavilla A, et al. Activity of cabazitaxel in castration-resistant prostate cancer progressing after docetaxel and next-generation endocrine agents. Eur Urol. 2014; 66: 459-465.

14. Wissing MD, Coenen JL, van den Berg P, et al. CAST: A retrospective analysis of cabazitaxel and abiraterone acetate sequential treatment in patients with metastatic castrate-resistant prostate cancer previously treated with docetaxel. Int J Cancer. 2015; 136: E760-772.

15. Al Nakouzi N, Le Moulec $\mathrm{S}$, Albiges $\mathrm{L}$, et al. Cabazitaxel Remains Active in Patients Progressing After Docetaxel Followed by Novel Androgen Receptor Pathway Targeted Therapies. Eur Urol. 2015; 68: 228-235.

16. Caffo O, De Giorgi U, Fratino L, et al. Clinical Outcomes of Castration-resistant Prostate Cancer Treatments Administered as Third or Fourth Line Following Failure of Docetaxel and Other Second-line Treatment: Results of an Italian Multicentre Study. Eur Urol. 2015; 68: 147-153.

17. Sonpavde G, Bhor M, Hennessy D, et al. Sequencing of Cabazitaxel and Abiraterone Acetate After Docetaxel in Metastatic Castration-Resistant Prostate Cancer: Treatment Patterns and Clinical Outcomes in Multicenter Community-Based US Oncology Practices. Clin Genitourin Cancer. 2015; 13: 309-318.

18. Angelergues A. Prognostic factors of survival in patients with metastatic castration resistant prostate cancer (mCRPC) treated with cabazitaxel: sequencing might matter. J Clin Oncol. 2013; 31 (Suppl. 6)
19. Maines F, Caffo O, Veccia A, et al. Sequencing new agents after docetaxel in patients with metastatic castration-resistant prostate cancer. Crit Rev Oncol Hematol. 2015; 96: 498-506.

20. van Soest RJ, de Morree ES, Kweldam CF, et al. Targeting the Androgen Receptor Confers In Vivo Cross-resistance Between Enzalutamide and Docetaxel, But Not Cabazitaxel, in Castration-resistant Prostate Cancer. Eur Urol. 2015; 67: 981-985.

21. Hofner T, Vallet S, Hadaschik BA, et al. Docetaxel followed by abiraterone in metastatic castration-resistant prostate cancer: efficacy and predictive parameters in a large single center cohort. World J Urol. 2015; 33: 833-839.

22. Buonerba C, Pond GR, Sonpavde G, et al. Potential value of Gleason score in predicting the benefit of cabazitaxel in metastatic castration-resistant prostate cancer. Future Oncol. 2013; 9: 889-897.

23. van Soest RJ, de Morree ES, Shen L, et al. Initial biopsy Gleason score as a predictive marker for survival benefit in patients with castration-resistant prostate cancer treated with docetaxel: data from the TAX327 study. Eur Urol. 2014; 66: 330-336.

24. Halabi S, Kelly WK, Ma H, et al. Meta-Analysis Evaluating the Impact of Site of Metastasis on Overall Survival in Men With Castration-Resistant Prostate Cancer. J Clin Oncol. 2016; 34: 1652-9.

25. Halabi S, Lin CY, Small EJ, et al. Prognostic model predicting metastatic castration-resistant prostate cancer survival in men treated with second-line chemotherapy. J Natl Cancer Inst. 2013; 105: 1729-1737.

26. Beltran H, Prandi D, Mosquera JM, et al. Divergent clonal evolution of castration-resistant neuroendocrine prostate cancer. Nat Med. 2016; 22: 298-305.

27. Gururajan M, Cavassani KA, Sievert $M$, et al. SRC family kinase FYN promotes the neuroendocrine phenotype and visceral metastasis in advanced prostate cancer. Oncotarget. 2015; 6: 44072-44083.

28. Sartor AO, Oudard S, Sengelov L, et al. Cabazitaxel vs docetaxel in chemotherapy-naive $(\mathrm{CN})$ patients with metastatic castration-resistant prostate cancer (mCRPC): A three-arm phase III study (FIRSTANA). J Clin Oncol. 2016; 34 (Suppl): Abstr5006.

29. De Bono JS, Hardy-Bessard AC, Kim CS, et al. Phase III non-inferiority study of cabazitaxel (C) $20 \mathrm{mg} / \mathrm{m} 2$ (C20) versus $25 \mathrm{mg} / \mathrm{m} 2$ (C25) in patients (pts) with metastatic castration-resistant prostate cancer (mCRPC) previously treated with docetaxel (D). J Clin Oncol. 2016; 34 (Suppl): Abstr5008. 